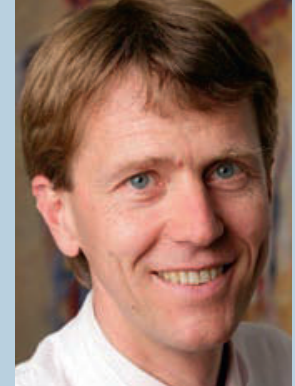

Prof. Dr. med. Heribert Schunkert Lübeck, Kardiologie

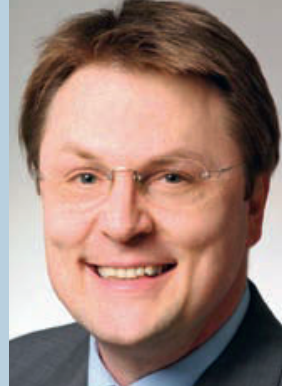

Prof. Dr. med. Jochen Seißler München, Diabetologie

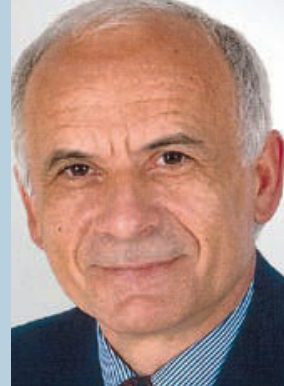

Prof. Dr. med. Alfred Wirth Bad Rothenfelde, Adipositas
Prof. Dr. med.

Walter Zidek

Berlin,

Hypertensiologie

\title{
Hypertonie
}

\section{Bessere Prognose bei initialer Kombinationstherapie}

Die Empfehlungen zur Hypertoniebehandlung beinhalten eine initiale Kombinationstherapie mit zwei Antihypertensiva. Es gibt aber wenig Daten darüber, wie eine initiale Kombinationstherapie die Prognose beeinflusst.

In einer Fallkontrollstudie wurde untersucht, mit welcher Häufigkeit kardiovaskuläre Ereignisse unter einer initialen Kombinationstherapie im Vergleich zu einer Monotherapie zu Beginn der Behandlung auftreten. Die Untersuchung basiert auf einer Kohorte von 209650 Patienten aus der Lombardei im Alter von 40-79 Jahren, die zwischen 2000 und 2001 erstmals mit Antihypertensiva behandelt wurden.

Als Fälle wurden 10688 Patienten definiert, die von Beginn der antihypertensiven Behandlung an bis 2007 wegen einer kardiovaskulären Erkrankung stationär aufgenommen wurden. Aus dem gesamten Kollektiv wurden für jeden dieser Fälle drei Kontrollen randomisiert ausgewählt. Die Fälle und die Kontrollpersonen wurden hinsichtlich kardiovaskulärer Ereignisse verglichen bei sofortiger Kombinationstherapie zu Beginn der Hochdruckbehandlung als auch bei einer anfänglichen Monotherapie.

Abbildung 1 zeigt, dass die initiale Kombinationstherapie signifikant weniger mit kardiovaskulären Ereignissen assoziiert war als der Beginn mit einer Monotherapie. Dies galt allerdings nur, wenn die Patienten auch im weiteren Verlauf eine Kombinationstherapie erhielten.

II Kommentar: Das Ergebnis ist interessant, weil hier ein Beleg dafür gewonnen wurde, dass die initiale Kombinationstherapie nicht nur für die Compliance der Patienten und für die rasche Blutdruckeinstellung wichtig ist, sondern offenbar auch mit weniger kardiovaskulären Ereignissen im Folgezeitraum verbunden ist.

Über die Ursachen hierfür kann man derzeit nur spekulieren. Die Autoren haben versucht, eine ganze Reihe von Confoundern (Begleitfaktoren, die das Ergebnis beeinflusst haben könnten) auszuschließen. Die klinischen Daten der Patienten sowie der sozio- ökonomische Status lieferten allerdings keine Erklärung für den unterschiedlichen Verlauf.

Auch wenn die Gründe für die unterschiedliche Prognose der Patientengruppen nicht kennen, liefern diese Daten doch weitere Argumente dafür, Hochdruckpatienten auch initial mit einer Kombinationstherapie zu behandeln.

Prof. Dr. med. Walter Zidek, Medizinische Klinik IV, Charité Berlin II

I Corrao G et al. Cardiovascular Protection by Initial and Subsequent Combination of Antihypertensive Drugs in Daily Life Practice. Hypertension 2011 (E-pub ahead of print)

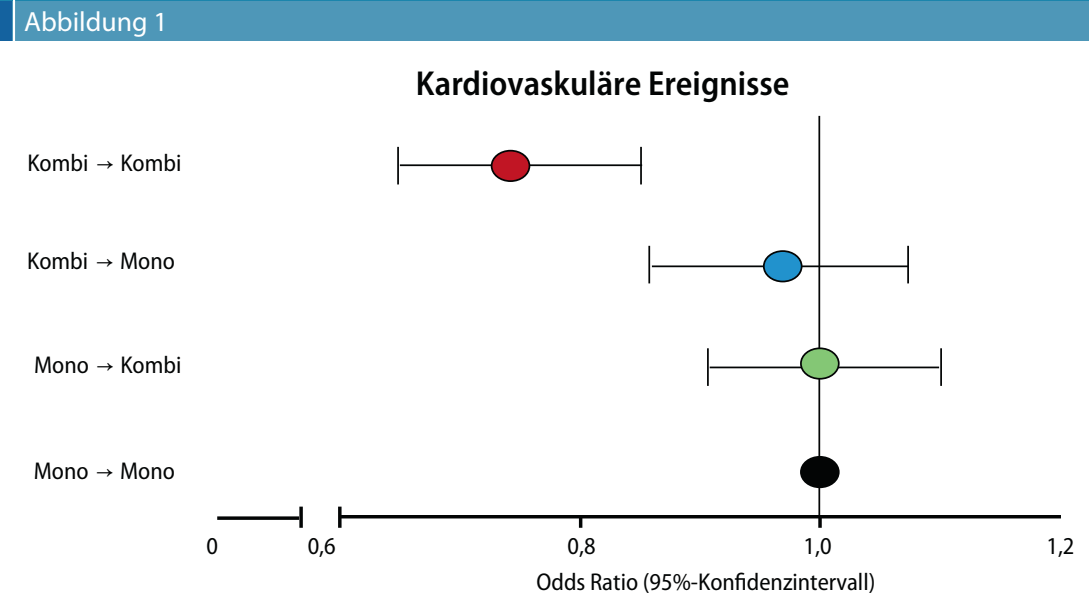

Effekt der initialen und der nachfolgenden Hypertonietherapie auf das kardiovaskuläre Risiko (zerebrovaskulär und koronar); Mono $=$ Monotherapie, $K o m b i=$ Kombinationstherapie. 